Original Research

\title{
"Real World" Australian Experience of Allogeneic Hematopoietic Stem Cell Transplantation (HSCT) in Adults with Severe Aplastic Anaemia.
}

\author{
Courtney Tate ${ }^{1,2,3}$, Jason P Butler ${ }^{1}$, Cameron Curley ${ }^{1}$, Siok-Keen Tey ${ }^{1,2,4}$, Glen A Kennedy ${ }^{1,2}$, \\ Ashleigh P Scott ${ }^{1,2, *}$
}

1. Royal Brisbane and Women's Hospital, Herston, QLD, Australia; E-mails: courtney.tate@health.qld.gov.au; Cameron.curley@health.qld.gov.au; jason.butler@health.qld.gov.au; glen.kennedy@health.qld.gov.au; Ashleigh.scott@health.qld.gov.au

2. The University of Queensland, St Lucia, QLD, Australia

3. Gold Coast University Hospital, Southport, QLD, Australia

4. QIMR Berghofer Medical Research Institute, Herston, QLD, Australia

* Correspondence: Ashleigh P Scott; E-Mail: Ashleigh.scott@health.qld.gov.au

Academic Editor: Martin Guimond

Special Issue: $\underline{\text { Allogeneic Stem Cell Transplantation }}$

OBM Transplantation

2020 , volume 4 , issue 4

doi:10.21926/obm.transplant.2004121
Received: June 23, 2020

Accepted: October 14, 2020

Published: October 23, 2020

\begin{abstract}
Acquired Severe Aplastic Anaemia (SAA) is a rare bone marrow failure syndrome, for which allogeneic haematopoietic stem cell transplant (HSCT) is a proven curative therapy. Despite excellent outcomes for matched sibling SAA recipients in terms of engraftment and survival, HSCT remains highly challenging in older matched-unrelated-donor (MUD) recipients, due to increased non-relapse mortality (NRM) from causes such as graft versus host disease (GVHD), organ failure and infection. We sought to evaluate our local HSCT outcomes for SAA, determine factors that predict for inferior outcomes, and benchmark local outcomes against international cohorts. To define outcomes for sibling and MUD HSCT in adults $\geq 17$ years of age with SAA at a single Australian institution between 2002 and 2018 and compare these outcomes to internationally published cohorts. The primary outcome was 1-year overall
\end{abstract}

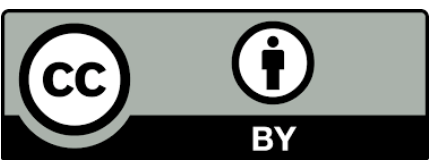

(C) 2020 by the author. This is an open access article distributed under the conditions of the Creative Commons by Attribution License, which permits unrestricted use, distribution, and reproduction in any medium or format, provided the original work is correctly cited. 
survival (OS). Secondary outcomes included the incidences of engraftment, response, secondary graft failure, GVHD, and moderate to severe organ dysfunction. All 21 patients comprising 10 sibling and 11 MUD HSCTs, with a median age of 30 (range 17-64), received bone marrow grafts. At a median follow up of 3.5 years, 17 (81\%) of 21 patients remained alive and in remission from SAA. 1-year OS, non-relapse mortality (NRM), and GVHD incidence were $85 \%, 15 \%$, and $48 \%$ respectively. Two patients died prior to engraftment at Day+27 and Day+33. When comparing sibling and MUD HSCT recipients, incidences of survival, engraftment and post-HSCT complications were similar; however, MUD HSCT survivors experienced a higher incidence of chronic GVHD (67\% vs. $11 \%$; $p=0.04)$. Age $>40$ years, AKI by Day+28, infection by Day +100 and deviation from intended GVHD prophylaxis predicted for worse OS. Our outcomes following HSCT for SAA are comparable to international cohorts with age $>40$ years, early onset infection, AKI and deviation from intended GVHD prophylaxis protocol negatively impacting OS. Further research is warranted to optimise MUD HSCT conditioning and GVHD prophylaxis protocols for SAA, particularly in older patients.

\section{Keywords}

Aplastic anaemia; HSCT; GVHD

\section{Introduction}

Acquired Severe Aplastic Anaemia (SAA) is a rare, life-threatening, immune-mediated acquired bone marrow failure syndrome. Hematopoietic stem cell transplantation (HSCT) is the preferred treatment in patients $<40$ years old with a matched sibling donor, as well as in patients with a fully matched unrelated donor (MUD) who have failed or relapsed despite prior immunosuppressive therapy (IST) [1].

HSCT conditioning protocols for matched sibling SAA recipients report excellent outcomes in terms of engraftment and survival; standard conditioning is cyclophosphamide (CY) $200 \mathrm{mg} / \mathrm{kg}$ (total) and horse (equine) anti-thymocyte globulin (ATG) $90 \mathrm{mg} / \mathrm{kg}$ (total) (CY-ATG). However, the optimal conditioning for MUD HSCT is less well established. Typical MUD conditioning incorporates low dose total body irradiation (TBI, 2Gy) (CY-ATG-TBI), which improves engraftment and overall survival (OS) for younger MUD recipients [2-4]. However, HSCT for SAA remains highly challenging in older MUD recipients, due to increased non-relapse mortality (NRM) from causes such as graft versus host disease (GVHD), organ failure and infection [4].

Recently, researchers have sought to reduce MUD HSCT NRM by modifying conditioning protocols and GVHD prophylaxis. Examples include T-cell manipulation, co-transplantation with mesenchymal stem cells, and dose-reducing or omitting high-dose cyclophosphamide and introducing fludarabine as a "cyclophosphamide-sparing" agent [5-9]. For matched adult HSCT using bone marrow as donor stem cell source, the most promising novel conditioning regimen, published by Anderlini et al [9], evaluated intermediate dose CY $50-100 \mathrm{mg} / \mathrm{kg}$ with rabbit ATG, fludarabine and TBI 2Gy (rATG-Cy50-100-Flu-TBI). Despite only $19 \%$ of the cohort being aged $>40$ years, moderate to severe organ dysfunction remains common before Day+100 (14\%), and graft 
failure and mortality are not insignificant (14\% and $16 \%$ respectively at 1 -year). For clinicians, performing MUD HSCT in SAA remains a complex juggling act between selecting conditioning strength to facilitate sustainable engraftment, optimising pre-HSCT patient medical selection and fitness to withstand conditioning, and delivering adequate GVHD prophylaxis.

Our institution uses CY-ATG-TBI (2Gy) as HSCT conditioning for patients aged up to 65 years undergoing MUD HSCT for SAA. To inform the design of a prospective clinical trial of a novel MUD HSCT protocol, we sought to evaluate our local HSCT outcomes for SAA using CT-ATG-TBI, and to explore the incidence and potential impact of complications such as GVHD, infection or organ failure.

\section{Materials and Methods}

\subsection{Study Population}

We conducted a retrospective analysis of all adults $\geq 17$ years undergoing HSCT for SAA at our institution between August 2002 and May 2018. The study was conducted in accordance with the Declaration of Helsinki and received appropriate local institutional approvals.

\subsection{Recipient Selection}

All HSCT recipients required a confirmed bone marrow diagnosis of SAA, and normal pre-HSCT organ function including normal echocardiogram ejection fraction (EF $>50 \%$ ), normal creatinine clearance and glomerular filtration rate (GFR $>60$ ), normal bilirubin and liver function tests (LFTs) and normal corrected pulmonary diffusion capacity (CDLCO $>65 \%$ ). No recipients were allowed to proceed to HSCT with an unresolved infection, and individualised transplant protocols including antibiotic prophylaxis and empiric antibiotic choice after accounting for prior infections were developed.

\subsection{Donor Selection}

All sibling donors were $8 / 8$ matched for HLA-A, -B, -C and DRB1 by serology typing for HLA class I antigens and allele typing using sequence specific oligonucleotide probes (SSOP) for HLA class II antigens. Serology typing was subsequently confirmed by SSOP. All unrelated donors were 10/10 high-resolution matches for HLA-A, -B, -C, DRB1 and DQ by allele typing using SSOP.

\subsection{HSCT Procedures}

All sibling HSCT recipients underwent CY-ATG conditioning, comprising cyclophosphamide $50 \mathrm{mg} / \mathrm{kg} /$ day on Day-5 to Day-2 (total $200 \mathrm{mg} / \mathrm{kg}$ ) and equine anti-thymocyte globulin (ATGAM, Pfizer Inc., New York, NY, USA) 30mg/kg/day (total $90 \mathrm{mg} / \mathrm{kg}$ ) on Day-5 to Day-3 (equine ATG was available throughout the study period). MUD HSCT recipients received $2 \mathrm{~Gy}$ TBI at Day-1 in addition to CY-ATG (CY-ATG-TBI). All patients received bone marrow grafts without in vitro T-cell depletion. Post-HSCT GVHD prophylaxis consisted of methotrexate with folinic acid rescue at $15 \mathrm{mg} / \mathrm{m}^{2}$ on Day +1 , and $10 \mathrm{mg} / \mathrm{m}^{2}$ on Days $+3,+6$ and +11 , as well as cyclosporin A (CsA) $5 \mathrm{mg} / \mathrm{kg}$ Day-1 to Day+1, and then $3 \mathrm{mg} / \mathrm{kg}$ from Day+2. CsA trough level targets were 150-300ug/L. CsA weaning was 
commenced on Day+180 and occurred over 3-6 months depending on individual patient chimerism and GVHD incidence. Tacrolimus was substituted in the event of CsA intolerance.

\subsection{Data Collection}

Patients were identified from an existing departmental database, and data were collected by retrospective chart review. Patients were divided into sibling and MUD HSCT cohorts for subsequent comparison.

\subsubsection{Baseline Patient Characteristics Collected}

-Patient demographics;

-Pre-HSCT organ function and prior significant infections;

-SAA details: severity, presence of associated paroxysmal nocturnal haemoglobinuria (PNH) clone at diagnosis, use of prior IST, time from diagnosis to HSCT;

-HSCT details: donor source, CMV matching, ABO matching, conditioning regimen, and infused stem cell number (total nucleated cell count, TNC).

\subsubsection{Post-HSCT Details}

-Engraftment details: day of neutrophil and platelet engraftment, incidence of primary and secondary graft failure, best SAA response;

-GVHD details: GVHD prophylaxis received, incidence and severity of acute and chronic GVHD $[10,11]$;

-Incidence of moderate to severe organ dysfunction (grade 2 to 5 as per CTCAE v4.03) including but not limited to: acute kidney injury (AKI) prior to Day+28, hyperbilirubinaemia, hepatitis, thrombotic microangiopathy (TMA), pulmonary haemorrhage, veno-occlusive disease (VOD), and infection prior to Day+100.

-Survival.

The primary outcome was 1-year OS. Secondary outcomes included the incidences of engraftment, response, secondary graft failure, GVHD, and moderate to severe organ dysfunction (grade 2 to 5 as per CTCAE v4.03).

\subsection{Statistical Analysis}

Outcomes between the two cohorts were compared using Chi-square test for categorical variables and Mann-Whitney test for continuous variables. OS was measured from time of stem cell infusion to date of death, or last date patient follow up at time of censoring. Survival curves were obtained using the Kaplan-Meier method and comparison performed using log-rank test. Univariate Cox proportional hazard model was used to analyse factors potentially impacting on overall survival including recipient age ( $<40$ vs. $\geq 40$ years), prior IST, number of lines of IST ( $<2$ vs. $\geq$ 2 ), days from diagnosis to HSCT (<6 months vs. $\geq 6$ months), AKI, infection, and GVHD. Results are expressed as hazard ratios (HR) with $95 \%$ confidence intervals $(\mathrm{Cl})$. GraphPad version 8.0.0 was used for statistical analysis. 


\section{Results}

\subsection{Baseline Characteristics}

21 patients were identified and included for analysis, comprising 10 sibling and 11 MUD HSCT. Donor and recipient characteristics are outlined in Table 1. Five (24\%) patients underwent first-line sibling HSCT. As expected, when comparing the MUD HSCT cohort with sibling HSCTs, there was a significantly greater time to HSCT, and higher incidence of prior IST use and ABO mismatch in the MUD HSCT cohort. In the sibling HSCT cohort there were more female donors due to selection preference of male donors for MUD HSCT when available.

Table 1 Donor, recipient and transplant characteristics.

\begin{tabular}{|c|c|c|c|c|}
\hline & All HSCTs & Sibling HSCTs & MUD HSCTS & p-value \\
\hline Transplant type & 21 & 10 & 11 & \\
\hline Bone marrow derived stem cells & 21 & 10 & 11 & \\
\hline Recipient age, median (range) & $30(17-64)$ & $26(17-58)$ & $31(18-64)$ & 0.29 \\
\hline Donor age, median (range) & $35(14-58)$ & $26(14-58)$ & $42(22-49)$ & 0.13 \\
\hline Male gender (\%) & $15(71)$ & $6(60)$ & $9(82)$ & 0.36 \\
\hline PNH clone ${ }^{\dagger}$ & $9(43)$ & $2(20)$ & $7(64)$ & 0.81 \\
\hline Prior IST $\ddagger$ n (\%) & $16(76)$ & $5(50)$ & $11(100)$ & $0.01 *$ \\
\hline No. treated with $\geq 2$ lines of IST & $4(19)$ & $0(0)$ & $4(36)$ & 0.09 \\
\hline \multicolumn{5}{|l|}{ Conditioning regimen§ } \\
\hline Cy/ATGAM/2Gy TBI, n (\%) & & & $11(100)$ & \\
\hline Cy/ATGAM, n (\%) & & $10(100)$ & & \\
\hline Days from diagnosis to HSCTף & $231(18-5185)$ & $86(18-436)$ & $310(124-5185)$ & $0.003^{*}$ \\
\hline $\begin{array}{l}\text { Donor female to male recipient, } \\
\mathrm{n}(\%)\end{array}$ & 4 (19) & $4(40)$ & $0(0)$ & $0.04 *$ \\
\hline \multicolumn{5}{|l|}{$A B O, n(\%)$} \\
\hline match & $11(52)$ & $8(80)$ & $3(27)$ & $0.03^{*}$ \\
\hline minor mismatch & $5(24)$ & $1(10)$ & $4(36)$ & \\
\hline major mismatch & $2(10)$ & $0(0)$ & $2(18)$ & \\
\hline
\end{tabular}


minor and major mismatch $\quad 3(14) \quad 1(10) \quad 2(18)$

Median infused cell dose, (range)

TNC, $\times 10^{8} / \mathrm{kg} ¥$

$\begin{array}{lll}2.51 & 1.975 & 2.66 \\ (1.15-23.94) & (1.5-11.03) & (1.15-23.94)\end{array}$

0.32

†PNH indicates paroxysmal nocturnal haemoglobinuria: The PNH clone in all patients was $<5 \%$, except for one patient where the PNH clone comprised $50 \%$ of granulocytes, $64 \%$ of monocytes, with $2 \%$ type III red cells. No patients had evidence of haemolysis; \#IST immunosuppressive therapy; §Conditioning regimens explained in detail in methods section; ๆ HSCT haematopoietic stem cell transplant; $¥$ TNC total nucleated cell

\subsection{Post-HSCT Outcomes}

Post-HSCT outcomes are summarised in Table 2. The median follow-up is 3.5 years (range 0.116 years).

Table 2 Haematopoietic Stem Cell Transplantation Outcomes.

\begin{tabular}{|c|c|c|c|c|}
\hline & $\begin{array}{l}\text { All } \\
\text { HSCTs' } \\
(n=21)\end{array}$ & $\begin{array}{l}\text { Sibling } \\
(n=10)\end{array}$ & $\begin{array}{l}\text { MUD } \\
(n=11)\end{array}$ & p-value \\
\hline Neutrophil engraftment, $\mathrm{n}(\%)$ & $19(91)$ & $9(90)$ & $10(91)$ & 0.83 \\
\hline Platelet engraftment, n (\%) & $19(91)$ & $9(90)$ & $10(91)$ & 0.10 \\
\hline $\begin{array}{l}\text { Deviation from intended GVHD§ prophylaxis, } n \\
\text { (\%) }\end{array}$ & $9(43)$ & $5(50)$ & $4(36)$ & 0.67 \\
\hline AKI prior to $\mathrm{D}+28, \mathrm{n}(\%)$ & $7(33)$ & $3(30)$ & $4(36)$ & $>0.99$ \\
\hline Infection prior to $\mathrm{D}+100, \mathrm{n}(\%)$ & $10(48)$ & $4(40)$ & $6(55)$ & 0.67 \\
\hline Any GVHD, n (\%) & $10(48)$ & $2(20)$ & $8(73)$ & $0.03 *$ \\
\hline Acute GVHD, n (\%) & $6(29)$ & $1(10)$ & $5(46)$ & 0.08 \\
\hline Chronic GVHD, n (\%) & $7(33)$ & $1(10)$ & $6(66)$ & $0.04^{*}$ \\
\hline - $\quad$ Extensive stage, $\mathrm{n}(\%)$ & $3(14)$ & $0(0)$ & $3(33)$ & 0.10 \\
\hline Mortality, n (\%) & $4(19)$ & $1(10)$ & $3(27)$ & 0.49 \\
\hline
\end{tabular}

†HSCT indicates haematopoietic stem cell transplant; ¥MUD matched unrelated donor; §GVHD graft versus host disease; AKI acute kidney injury 


\subsubsection{Engraftment and Response}

Two (10\%) patients, comprising one sibling and one MUD HSCT recipient, died at Day+27 and Day+33 respectively, prior to neutrophil engraftment. In those who did engraft, sibling and MUD cohorts showed similar median time to engraftment for neutrophils (18 [range 16-23] vs. 20 [range 12-46] days respectively; $p=0.83$ ) and platelets ( 32 [range 24-312] vs. 25 [range 21-56] days respectively; $p=0.10$ ).

All those who engrafted achieved a complete response (CR) from their SAA. There were no cases of secondary graft failure. One patient had mixed chimerism and refractory thrombocytopenia at Day+180 despite full morphological megakaryocyte engraftment on bone marrow aspirate, indicating a degree of peripheral platelet consumption. He underwent second HSC infusion from the same sibling donor, which was unsuccessful, but ultimately achieved a normal platelet count after therapeutic splenectomy despite ongoing mixed chimerism; thus consistent with a diagnosis of post-HSCT immune thrombocytopenic purpura.

\subsubsection{Incidence of Moderate-Severe Organ Dysfunction}

13 patients (62\%) experienced at least one episode of moderate-severe organ dysfunction prior to Day+100 post HSCT, described in Table 3. These included infection (10), AKI (7), hepatic dysfunction (3), heart failure (1), pulmonary haemorrhage (1; secondary to infection), and thrombotic microangiopathy (TMA) (1). There were no cases of VOD. The incidence of any moderate-severe organ dysfunction did not differ between sibling and MUD recipients (60\% vs $64 \% ; p=1.0$ ), however, the incidence was non-significantly higher in those aged $>40$ years compared to $<40$ years ( $83 \%$ vs $53 \%$; $p=0.33$ ).

9 of these patients (43\% of the entire cohort) experienced their episode(s) of acute moderatesevere organ dysfunction within the first 14 days post HSCT, and consequently their intended GVHD prophylaxis was modified or temporarily omitted until resolution of the organ dysfunction (Table 3). Reasons for deviation included grade 2-3 AKI $(n=5)$, grade 3-4 hyperbilirubinaemia $(n=2)$ or transaminitis $(n=1)$, and grade 3 TMA $(n=1)$. Rates of deviation from intended GVHD prophylaxis were similar between sibling and MUD HSCT cohorts ( $50 \%$ vs. $36 \% ; p=0.67$ ), between patients $\geq 40$ and $<40$ years ( $50 \%$ vs. $67 \% ; p=0.98)$ and between patients who received upfront HSCT compared with prior IST ( $60 \%$ vs. $38 \%$; $p=0.61)$. 
OBM Transplantation 2020; 4(4), doi:10.21926/obm.transplant.2004121

Table 3 Modifications to graft versus host disease (GVHD) prophylaxis and patient outcomes.

\begin{tabular}{|c|c|c|c|c|c|c|c|c|}
\hline $\begin{array}{l}\text { Recipient } \\
\text { Age/sex }\end{array}$ & Sib/MUD & $\begin{array}{l}\text { Intended GVHD } \\
\text { prophylaxis }\end{array}$ & $\begin{array}{l}\text { Indication for altering } \\
\text { GVHD prophylaxis** }\end{array}$ & $\begin{array}{l}\text { Modification to } \\
\text { GVHD prophylaxis }\end{array}$ & $\begin{array}{l}\text { D100 } \\
\text { Infection }\end{array}$ & $\begin{array}{l}\text { Acute } \\
\text { GVHD } \\
\text { Grade }\end{array}$ & $\begin{array}{l}\text { Chronic GVHD } \\
\text { Seattle stage }\end{array}$ & $\begin{array}{l}\text { Outcome/days post } \\
\text { HSCT at censoring }\end{array}$ \\
\hline $24 \mathrm{~F}$ & $\mathrm{sib}$ & $\mathrm{CsA}^{*}, \mathrm{MTX}$ & Grade 2 AKI D+5 & $\begin{array}{l}\text { Tacro from } D+5 \\
\text { instead of CsA }\end{array}$ & $N$ & 0 & 0 & 4562 \\
\hline $23 \mathrm{M}$ & sib & CsA*, MTX & $\begin{array}{l}\text { Grade } 3 \\
\text { hyperbilirubinaemia } \\
D+13\end{array}$ & $\begin{array}{l}\text { MMF from } D+13 \\
\text { instead of } C s A\end{array}$ & $Y$ & 0 & $\begin{array}{l}\text { Limited } \\
\text { (cutaneous) }\end{array}$ & 1249 \\
\hline $17 \mathrm{M}$ & $\mathrm{sib}$ & CsA, MTX & NA & NA & $\mathrm{N}$ & 0 & 0 & 1537 \\
\hline $19 M$ & $\mathrm{sib}$ & CsA, MTX & NA & NA & $\mathrm{N}$ & 0 & 0 & 1467 \\
\hline $58 \mathrm{~F}$ & $\mathrm{sib}$ & CsA, MTX & NA & NA & $Y$ & II & 0 & 629 \\
\hline $28 \mathrm{~F}$ & sib & CsA, MTX & NA & NA & $N$ & 0 & 0 & 553 \\
\hline $32 M$ & sib & CsA, MTX & NA & NA & $\mathrm{N}$ & 1 & 0 & 379 \\
\hline $36 \mathrm{M}$ & sib & CsA, MTX* & Grade 2 AKI D+12 & $\begin{array}{l}\text { D+11 MTX } \\
\text { withheld, MP } 1 \\
\mathrm{mg} / \mathrm{kg} \text { until CsA } \\
\text { therapeutic, and } \\
\text { Basiliximab }\end{array}$ & $Y$ & 0 & 0 & 188 \\
\hline $20 \mathrm{M}$ & $\mathrm{sib}$ & CsA, MTX* & $\begin{array}{l}\text { Grade } 4 \text { transaminitis } \\
D+3\end{array}$ & $\begin{array}{l}\mathrm{D}+3,+6,+11 \mathrm{MTX} \\
\text { withheld }\end{array}$ & $\mathrm{N}$ & 0 & 0 & 116 \\
\hline
\end{tabular}




\begin{tabular}{|c|c|c|c|c|c|c|c|c|}
\hline & & & & $\begin{array}{l}\text { CsA stopped } D+3 \text {, } \\
D+6 \text { MTX dose }\end{array}$ & & & & $\begin{array}{l}\text { Death } D+27 \text { : cyclo- } \\
\text { phosphamide }\end{array}$ \\
\hline $49 \mathrm{~F}$ & sib & CsA*, MTX* & Grade 2 AKI D+3 & $\begin{array}{l}\text { reduced to } 9 \mathrm{mg} \text {, } \\
\mathrm{D}+11 \\
\text { withheld }\end{array}$ & $Y$ & NA & NA & $\begin{array}{l}\text { induced cardio- } \\
\text { myopathy with } \\
\text { respiratory failure }\end{array}$ \\
\hline $31 \mathrm{M}$ & MUD & CsA, MTX & NA & NA & $Y$ & II & $\begin{array}{l}\text { extensive } \\
\text { (myalgias, } \\
\text { arthralgias) }\end{array}$ & 4427 \\
\hline $18 \mathrm{~F}$ & MUD & Tacro*, MTX* & $\begin{array}{l}\text { Grade } 3 \text { Tacrolimus- } \\
\text { related TMA D+5 }\end{array}$ & $\begin{array}{lr}\text { D+5 MP } 1 & \mathrm{mg} / \mathrm{kg} \\
\text { and MMF } & 1 \mathrm{~g} \text { bd } \\
\text { instead } & \text { of } \\
\text { Tacrolimus } & \text { and } \\
\begin{array}{ll}\text { D+6, +11 } \\
\text { withheld }\end{array} & \end{array}$ & $\mathrm{N}$ & 0 & $\begin{array}{l}\text { extensive } \\
\text { (cutaneous) }\end{array}$ & 4382 \\
\hline $20 \mathrm{M}$ & MUD & CsA, MTX & NA & NA & $\mathrm{N}$ & 0 & $\begin{array}{l}\text { limited } \\
\text { (cutaneous) }\end{array}$ & 3256 \\
\hline $37 \mathrm{M}$ & MUD & CsA, MTX & NA & NA & $Y$ & I & 0 & 3290 \\
\hline $50 \mathrm{M}$ & MUD & CsA, MTX & NA & NA & $Y$ & III & 0 & 1756 \\
\hline $24 M$ & MUD & CsA, MTX & NA & NA & $\mathrm{N}$ & 0 & 0 & 1454 \\
\hline $28 \mathrm{~F}$ & MUD & CsA, MTX & NA & NA & $\mathrm{N}$ & 0 & $\begin{array}{l}\text { limited } \\
\text { (cutaneous) }\end{array}$ & 1454 \\
\hline $41 \mathrm{M}$ & MUD & CsA, MTX & NA & NA & $\mathrm{N}$ & II & $\begin{array}{l}\text { limited } \\
\text { (cutaneous) }\end{array}$ & 334 \\
\hline
\end{tabular}




\begin{tabular}{|c|c|c|c|c|c|c|c|c|}
\hline $64 \mathrm{M}$ & MUD & $\mathrm{CsA}^{*}, \mathrm{MTX}^{*}$ & Grade 2 AKI D+5 & $\begin{array}{l}\mathrm{D}+1,+3+6+11 \\
\mathrm{MTX} \text { withheld, } \\
\mathrm{MMF} 1 \text { bd and } \\
\text { prednisolone } 1 \\
\mathrm{mg} / \mathrm{kg} \text { from } \mathrm{D}+3\end{array}$ & Y & II & $\begin{array}{l}\text { extensive } \\
\text { (cutaneous) }\end{array}$ & $\begin{array}{l}\text { Death D+1350: RSV } \\
\text { pneumonitis with } \\
\text { respiratory failure }\end{array}$ \\
\hline $30 \mathrm{M}$ & MUD & CsA*, MTX* & Grade 2 AKI D+12 & 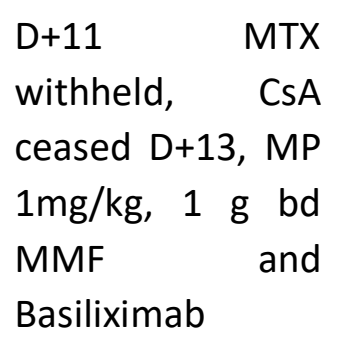 & $\mathrm{Y}$ & IV & NA & $\begin{array}{l}\text { Death D+102: acute } \\
\text { lower gastro- } \\
\text { intestinal GVHD }\end{array}$ \\
\hline $63 \mathrm{M}$ & MUD & Tacro, MTX* & $\begin{array}{l}\text { Grade } 3 \\
\text { hyperbilirubinaemia } \\
D+3\end{array}$ & $\begin{array}{l}D+11 \\
\text { withheld }\end{array}$ & Y & NA & NA & 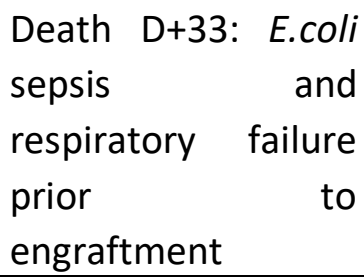 \\
\hline
\end{tabular}

*indicates modified GVHD prophylaxis; ${ }^{* *}$ Grading as per CTCAE v4.03 Sib: sibling; MUD: matched unrelated donor; CsA: Cyclosporin A; MTX: methotrexate; Tacro: Tacrolimus; D: Day; AKI: acute kidney injury; MMF: mycophenolate mofetil; MP: methylprednisone; TMA: thrombotic microangiopathic anaemia. 


\subsubsection{GVHD Details}

The incidences of acute and chronic GVHD are shown in Figure 1. In patients who engrafted $(n=19)$, there was a non-significant trend towards a higher incidence of moderate to severe (Grade II-IV) acute GVHD in MUD compared to sibling HSCT ( $50 \%$ vs. $11 \%, p=0.08$ ). Severe (Grade III-IV) acute GVHD was seen in MUD HSCT only (20\%). In those surviving beyond Day+100 (18 patients, comprising nine sibling and nine MUD HSCT), chronic GVHD occurred in seven (39\%) patients. Chronic GVHD occurred significantly more frequently in MUD compared to sibling HSCT (67\% vs. $11 \%, p=0.04)$. Two of the three cases of extensive chronic GVHD occurred in MUD HSCT, and two of these patients had modifications to their GVHD prophylaxis (Table 3). The incidence of GVHD was similar between patients with unmodified compared to modified GVHD prophylaxis (60\% vs. $55 \% ; p=0.96)$.
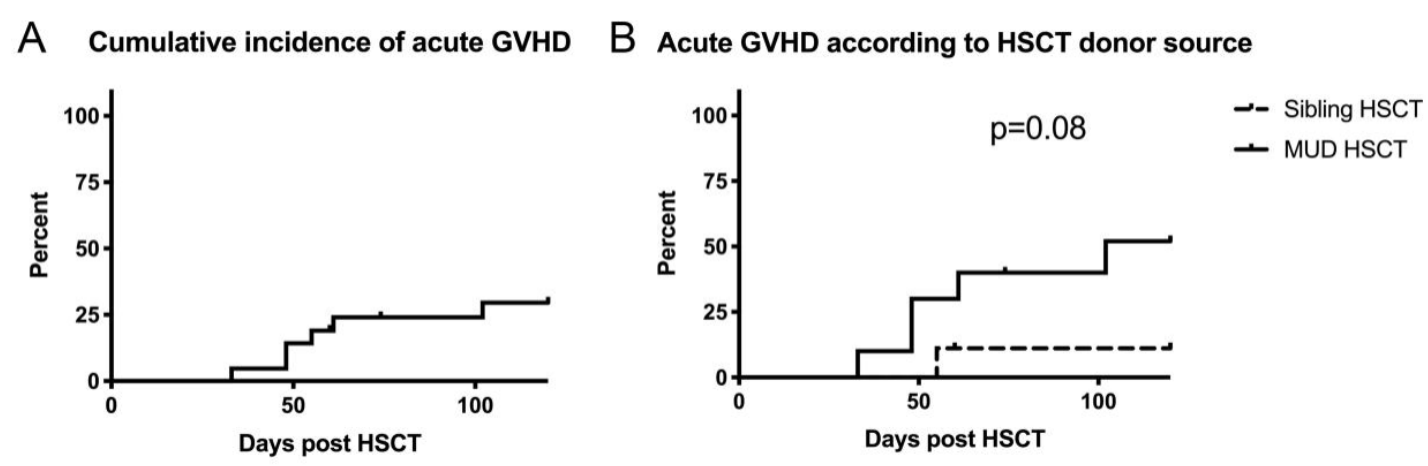

C Cumulative incidence of chronic GVHD
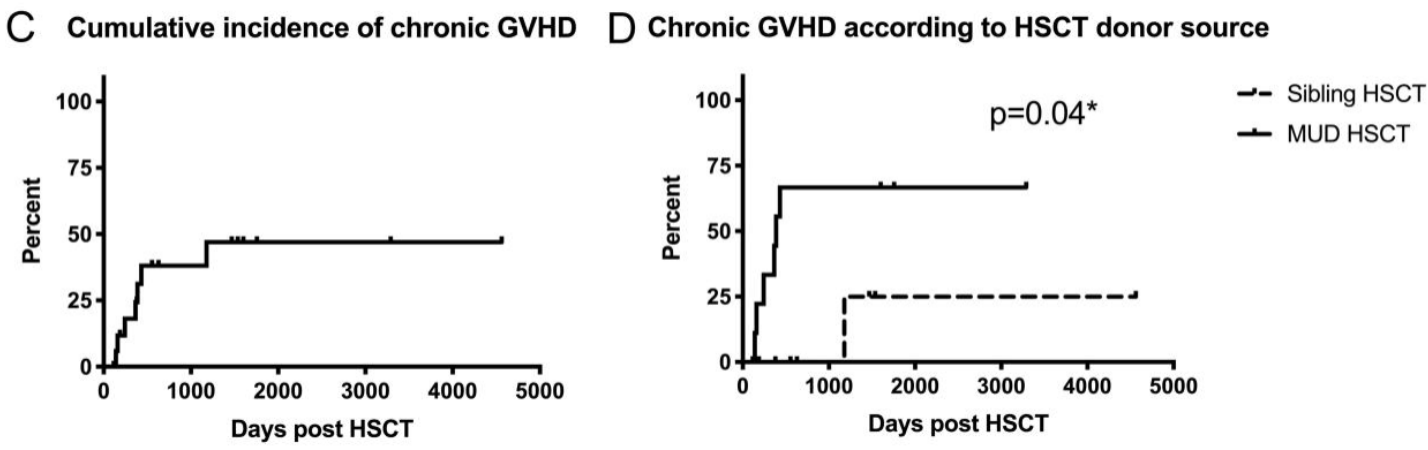

Figure 1 Incidence of graft versus host disease (GVHD) post allograft for severe aplastic anaemia (SAA). A. Cumulative incidence of acute GVHD. B. Acute GVHD according to hematopoietic stem cell transplant (HSCT) donor source. C. Cumulative incidence of chronic GVHD. D. Chronic GVHD according to HSCT donor source. MUD; matched unrelated donor.

\subsubsection{Survival}

At a median follow up of 3.5 years, 17 (81\%) HSCT recipients remain alive and in remission from SAA, with NRM $19 \%$ and death due to secondary graft failure $0 \%$. There was no significant difference in OS between sibling and MUD cohorts at 1 year (1-year OS 90\% vs. 82\% respectively; $\mathrm{p}=0.93$ ) (Figure 2 ) or at the time of censoring (OS $90 \%$ vs. $71 \%$ respectively; $p=0.48$ ). Notably, at the time of censoring, survival in the 6 patients aged $>40$ years is $50 \%$. Of those who had experienced early AKI but survived beyond $D+100$, none developed chronic kidney disease. 


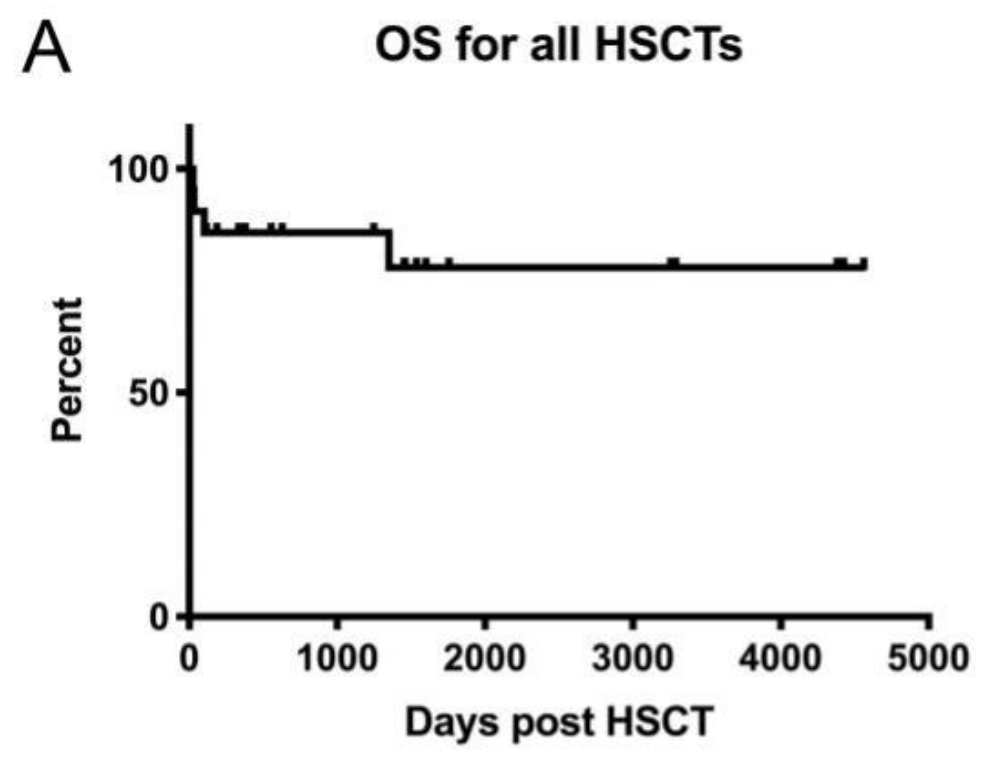

\section{B OS according to HSCT donor source}

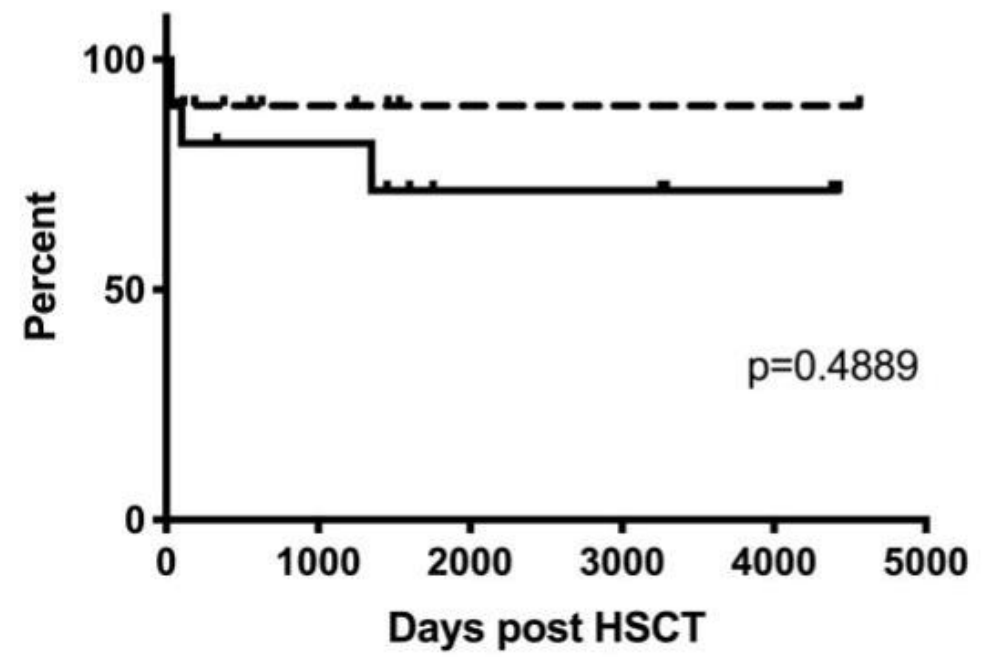

Figure 2 Overall survival (OS) post allograft for severe aplastic anaemia (SAA). A. OS for all allografts performed for SAA. B. OS according to hematopoietic stem cell transplant (HSCT) donor source. MUD; matched unrelated donor.

MUD mortality occurred secondary to refractory acute GVHD at Day+102 $(n=1)$, sepsis and secondary respiratory failure in the setting of primary graft failure at Day+33 $(n=1)$, and respiratory syncytial virus associated pneumonitis and respiratory failure in the setting of longterm immunosuppression for extensive cutaneous GVHD at 4 years $(n=1)$. One sibling HSCT recipient died at Day+27 due to cyclophosphamide induced cardiomyopathy and secondary respiratory failure prior to engraftment. All patients who died had required protocol deviations from planned GVHD prophylaxis due to early complications, compared with no deaths in patients who did not require protocol deviations ( $44 \%$ vs. $0 \%$ respectively; $p=0.09$ ). 


\subsection{Univariate Analysis}

Univariate analysis identified age $>40$ years (HR 15.4; 95\% $\mathrm{Cl} 1.6-151.1 ; \mathrm{p}=0.02)$, AKI (HR 77.31; 95\% Cl 7.5-796.0; $p=0.0005$ ), infection ( $\mathrm{HR} 9.137 ; 95 \% \mathrm{Cl} 1.2-65.7 ; p=0.03$ ), and deviation from intended GVHD prophylaxis (HR 15.84; $95 \% \mathrm{Cl} 2.0-12.8 ; \mathrm{p}=0.008$ ) as predictors of adverse OS in the total cohort. There was a non-significant trend toward inferior OS if the time from initial SAA diagnosis to HSCT exceeded 6 months, compared with those transplanted within 6 months of initial diagnosis (OS $100 \%$ vs. $67 \%$; $p=0.08$ ). Multivariate analysis was not performed due to small study cohort size.

\section{Discussion}

This is the largest case series of adult allogeneic HSCT undertaken for SAA in Australia and complements our previous analyses of SAA outcomes including allograft and non-allograft patients $[12,13]$. Although these retrospective data are underpowered compared to those published by the EBMT and CIBMTR, they do provide valuable insight into HSCT for SAA practice in Australia.

Compared to post-HSCT survival for malignancies such as leukaemia, SAA HSCT survival outcomes typically reflect NRM rather than post-HSCT relapse or secondary graft failure. We have previously described 15 patients transplanted at this institution for SAA between 1989-1999 with $100 \% 6$-year OS [12]. Notably, this cohort was significantly younger (median age 22 years, range 346) than the current cohort (median age 30 years, range 18-64), reflecting changes in transplantation practice over decades.

In this current cohort (2002-2018), we report 81\% OS and 19\% NRM at median 3.5 years followup. Although 1-year OS was similar between sibling and MUD recipients ( $90 \%$ vs. $82 \%$; $p=0.1$ ), we note that 3 of 4 deaths occurred in MUD HSCT patients, and thus likely reflects the known association between MUD HSCT and increased NRM. Not unexpectedly, compared to sibling HSCT, MUD HSCT survivors experienced a significantly greater incidence of chronic GVHD and a nonsignificant trend toward an increased incidence of acute GVHD. Overall, local outcomes for survival, engraftment and acute GVHD for sibling and MUD HSCT appear similar to those reported in large international series [2, 3].

Interestingly, we report a higher incidence of chronic GVHD (67\%; predominantly cutaneous) in MUD recipients than that published elsewhere (14-44\%) [2, 3]. Potential contributors include: a) the overall older age of our MUD population (median 35 years), and b) a potential centre effect, as chronic GVHD incidence (particularly cutaneous) at our institution is typically high compared to other international centres across all disease groups, occurring in $>50 \%$ of HSCT survivors beyond 6 months of follow up. Any centre effect for chronic GVHD incidence may be magnified by the low incidence of relapse for SAA compared to other malignant indications for HSCT.

Most notably, our study highlights an apparently substantial incidence of unexpected moderate-severe organ dysfunction ( $43 \%$ prior to $D+14,62 \%$ prior to $D+100$ ), occurring despite rigorous pre-HSCT evaluation of organ function and use of standard HSCT conditioning. When these complications occurred prior to $D+14$, the impact appeared substantial: all required dose reduction or omission of GVHD prophylaxis, with a possible negative impact upon survival. AKI was the major contributor in half of these cases prior to D+14 (24\% of the entire cohort); conversely, AKI occurring after engraftment but prior to $D+100(n=2)$ did not impact survival. We have 
previously reported in non-SAA HSCT that early AKI significantly increases post-HSCT NRM due to an increased incidence of acute and chronic GVHD [14]. Potentially, a significant proportion of NRM in SAA HSCT may be either attributable to direct toxicity from HSCT conditioning, or indirect toxicity due to subsequent GVHD prophylaxis modification resulting in fatal GVHD.

Our study reinforces a pertinent issue for SAA HSCT: that older MUD ( $>40$ years) recipients have relatively poor post-HSCT OS due to excess NRM $[6,15]$. In our series, OS for those aged $>40$ years at HSCT was $50 \%(n=6)$, including two who died of cardiac and pulmonary toxicities prior to engraftment, and one from complications of GVHD. All three remaining survivors $>40$ years experienced moderate to severe chronic GVHD. Furthermore, although our study is not powered to fully evaluate risk factors for organ dysfunction or any impact upon survival, the highest incidence was observed in MUD HSCT recipients aged $>40$ years.

Our study's results support research investigating novel MUD HSCT conditioning strategies that aim to minimise toxicity and therefore NRM. Although the studies reported to date are highly informative and valuable, patients aged $>40$ years appear relatively under-reported, likely reflecting a) the rarity of SAA overall, b) the relative infrequency of HSCT in this age group, and c) compared to younger SAA patients, the increased likelihood that older patients will have comorbidities that preclude their enrolment in HSCT trials. For MUD recipients aged $>40$ years, it is not yet clear which, if any, conditioning protocol is safest. Whilst we acknowledge the inherent limitations of comparing our small retrospective study with a prospective phase II trial, the incidence of grade 3-5 organ dysfunction following CY-ATG-TBI in our study appears similar to that reported following rATG-Cy50-100-Flu-TBI (27\% vs $22 \%$ ). This is apparently despite our MUD recipients being aged $>40$ years in $36 \%$ and $18-40$ years in $64 \%$, compared with $21 \%$ and $41 \%$ respectively [9]. Currently, for MUD HSCT recipients aged $>40$ years, alternative HSCT protocols lack convincing evidence for superior safety compared to existing regimens.

The authors acknowledge the major limitations of this study are its retrospective design and small numbers which limit analysis of prognostic factors that occur at a low incidence; however, this is not dissimilar to many other SAA HSCT publications due to the rarity of the disease.

\section{Conclusion}

Our single-centre outcomes following sibling or MUD HSCT for SAA appear comparable to those in the published literature, with the exception of higher rates of chronic cutaneous GVHD which may reflect the older age of our cohort. Despite acceptable OS, HSCT for SAA remains challenging due to a high incidence of early organ dysfunction, particularly AKI, which likely contribute to increased NRM by necessitating deviations from planned GVHD prophylaxis. Further research is warranted to optimise MUD HSCT conditioning and GVHD prophylaxis protocols for SAA, particularly in older patients.

\section{Acknowledgements}

The authors wish to thank Dr Andrea S Henden, Dr A James Morton, Dr Elango Subramoniapillai and Dr Kirk Morris for review of the manuscript. 


\section{Author Contributions}

All authors contributed to study concept and design, and contributed to the manuscript. CT and APS collected and analysed the data, and wrote the first and final drafts of the manuscript. CT, APS and JPB performed the statistical analyses.

\section{Competing Interests}

The authors have declared that no competing interests exist.

\section{Reference}

1. Bacigalupo A. How I treat acquired aplastic anemia. Blood. 2017; 129: 1428-1436.

2. Bacigalupo A, Socie G, Hamladji RM, Aljurf M, Maschan A, Kyrcz-Krzemien S, et al. Current outcome of HLA identical sibling versus unrelated donor transplants in severe aplastic anemia: An EBMT analysis. Haematologica. 2015; 100: 696-702.

3. Champlin RE, Perez WS, Passweg JR, Klein JP, Camitta BM, Gluckman E, et al. Bone marrow transplantation for severe aplastic anemia: A randomized controlled study of conditioning regimens. Blood. 2007; 109: 4582-4585.

4. Deeg HJ, O'Donnell M, Tolar J, Agarwal R, Harris RE, Feig SA, et al. Optimization of conditioning for marrow transplantation from unrelated donors for patients with aplastic anemia after failure of immunosuppressive therapy. Blood. 2006; 108: 1485-1491.

5. Bacigalupo A, Locatelli F, Lanino E, Marsh J, Socie G, Maury S, et al. Fludarabine, cyclophosphamide and anti-thymocyte globulin for alternative donor transplants in acquired severe aplastic anemia: A report from the EBMT-SAA working party. Bone Marrow Transplant. 2005; 36: 947-950.

6. Giammarco S, Peffault de Latour R, Sica S, Dufour C, Socie G, Passweg J, et al. Transplant outcome for patients with acquired aplastic anemia over the age of 40: Has the outcome improved? Blood. 2018; 131: 1989-1992.

7. Maury S, Bacigalupo A, Anderlini P, Aljurf M, Marsh J, Socie G, et al. Improved outcome of patients older than 30 years receiving HLA-identical sibling hematopoietic stem cell transplantation for severe acquired aplastic anemia using fludarabine-based conditioning: A comparison with conventional conditioning regimen. Haematologica. 2009; 94: 1312-1315.

8. Maury S, Balere-Appert ML, Pollichieni S, Oneto R, Yakoub-Agha I, Locatelli F, et al. Outcome of patients activating an unrelated donor search for severe acquired aplastic anemia. Am J Hematol. 2013; 88: 868-873.

9. Anderlini P, Wu J, Gersten I, Ewell M, Tolar J, Antin JH, et al. Cyclophosphamide conditioning in patients with severe aplastic anaemia given unrelated marrow transplantation: A phase 1-2 dose de-escalation study. Lancet Haematol. 2015; 2: e367-e375.

10. Filipovich AH, Weisdorf D, Pavletic S, Socie G, Wingard JR, Lee SJ, et al. National institutes of health consensus development project on criteria for clinical trials in chronic graft-versus-host disease: I. Diagnosis and staging working group report. Biol Blood Marrow Transplant. 2005; 11: 945-956.

11. Przepiorka D, Weisdorf D, Martin P, Klingemann HG, Beatty P, Hows J, et al. 1994 consensus conference on acute GVHD grading. Bone Marrow Transplant. 1995; 15: 825-828. 
12. Mollee P, Woodward N, Durrant S, Lockwood L, Gillett EA, Morton J, et al. Single institution outcomes of treatment of severe aplastic anaemia. Intern Med J. 2001; 31: 337-342.

13. Scott A, Morris K, Butler J, Mills AK, Kennedy GA. Treatment of aplastic anaemia with lowerdose anti-thymocyte globulin produces similar response rates and survival as per standard dose anti-thymocyte globulin schedules. Intern Med J. 2016; 46: 1198-1203.

14. Birchley A, Butler JP, Scott A, Curley C, Kennedy G. Early acute kidney injury post allogeneic hematopoetic progenitor cell transplant is associated with increased risk of GVHD, higher mortality and reduced survival. Blood. 2016; 128: 2227.

15. Gupta V, Eapen M, Brazauskas R, Carreras J, Aljurf M, Gale RP, et al. Impact of age on outcomes after bone marrow transplantation for acquired aplastic anemia using HLA-matched sibling donors. Haematologica. 2010; 95: 2119-2125.

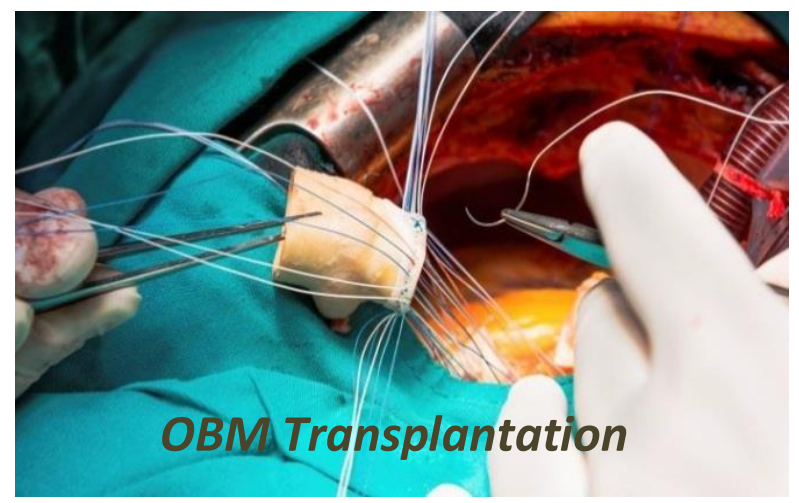

Enjoy OBM Transplantation by:

1. Submitting a manuscript

2. Joining in volunteer reviewer bank

3. Joining Editorial Board

4. Guest editing a special issue

For more details, please visit:

http://www.lidsen.com/journals/transplantation 